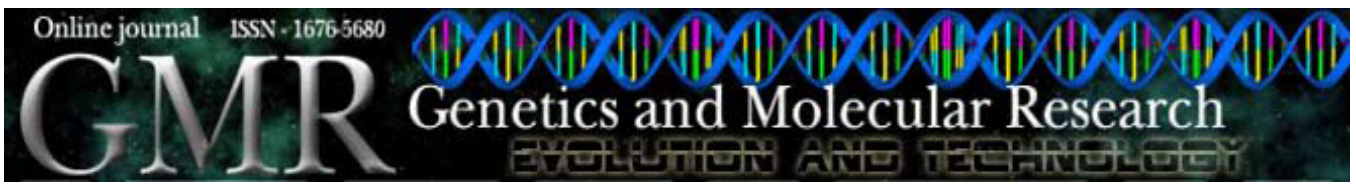

\title{
Acetaldehyde dehydrogenase 2 SNP rs671 and susceptibility to essential hypertension in Mongolians: a case control study
}

\author{
T. Hasi, L. Hao, L. Yang and X.L. Su \\ Clinical Research Center of Affiliated Hospital, Department of Surgery, \\ Inner Mongolian Medical College, Hohhot, Inner Mongolia, China \\ Correspondence author: X.L. Su \\ E-mail: xlsu@hotmail.com
}

Genet. Mol. Res. 10 (1): 537-543 (2011)

Received September 8, 2010

Accepted November 3, 2010

Published March 29, 2011

DOI 10.4238/vol10-1gmr1056

\begin{abstract}
Mongolians are known as heavy drinkers, and they have a high incidence of essential hypertension, which may be an associated pathology. We examined a possible association of essential hypertension and polymorphism of the aldehyde dehydrogenase 2 (ALDH2) gene in Mongolians from Inner Mongolia. Single nucleotide polymorphism rs671 of ALDH2 was detected by TaqMan PCR in 91 essential hypertensive patients (44 males and 47 females) and 70 healthy Mongolians (37 males and 33 females). Frequencies of the ALDH2*1/1 genotype and the ALDH2* 1 allele in patients (91.2 and $95.6 \%$, respectively) were significantly higher than in controls (78.6 and $89.3 \%$; $\mathrm{P}<0.05)$, while frequencies of ALDH $2 * 1 / 2$ genotype and ALDH2*2 allele in patients $(8.79$ and $4.4 \%)$ were much lower than in controls $(21.4$ and $10.7 \%$; $<<0.05)$. Frequencies of ALDH $2 * 1 / 1$ genotype and ALDH $2 * 1$ allele in female patients (95.8 and 94.9\%) were higher than in female controls $(70.0$
\end{abstract}


and $84.9 \% ; \mathrm{P}<0.05)$; frequencies of the ALDH $2 * 1 / 2$ genotype and the ALDH $2 * 2$ allele in female patients $(4.25$ and $2.13 \%)$ were lower than in female controls $(30.3$ and $15.2 \%, \mathrm{P}<0.05)$. There was no significant difference in male subjects. ALDH $2 * 2 / 2$ was not found in any of the subjects. We conclude that ALDH2 polymorphism is associated with essential hypertension in Mongolians, especially in female Mongolians. ALDH2*2 was found to be a negative risk factor for essential hypertension in Mongolians from Inner Mongolia.

Key words: Aldehyde dehydrogenase 2; Single nucleotide polymorphism; Mongolian nationality; Essential hypertension

\section{INTRODUCTION}

Essential hypertension $(\mathrm{EH})$ is a chronic disease. Interaction of multiple environmental and genetic factors is involved in EH. EH patients with different genetic backgrounds may have various responses to environmental factors (Yamamoto et al., 1993).

Previous studies showed that alcohol consumption is associated with blood pressure and is a risk factor for hypertension (Beilin, 1995; Chen et al., 2008; TsuchihashiMakaya et al., 2009). Aldehyde dehydrogenase 2 (ALDH2) is a crucial enzyme in alcohol metabolism. ALDH2, whose gene is located on chromosome 12, oxidizes aldehyde to acetic acid mainly in the mitochondria. There is a mutation in exon 12 with $\mathrm{G} \rightarrow \mathrm{A}$ substitution (single nucleotide polymorphism, SNP rs671, Glu487Lys) that inactivates the enzyme activity (Ferguson and Goldberg, 1997). Wild-type ALDH2 (ALDH2*1/1) works more efficiently in alcohol metabolism than heterozygous ALDH (ALDH2*1/2) and homozygous mutant $(\mathrm{ALDH} 2 * 2 / 2)$. It is reported that the concentration of aldehyde after alcohol consumption in individuals with ALDH2*2/2 is three and nineteen times higher than in individuals with ALDH2*1/2 and ALDH2*1/1, respectively (Yamamoto et al., 1993). ALDH2*2/2 homozygotes experience facial flushing, headache, nausea, and other adverse symptoms, and therefore, ALDH $2 * 2 / 2$ homozygotes can tolerate less alcohol than ALDH $2 * 1 / 1$ homozygotes and ALDH $2 * 1 / 2$ heterozygotes. Additionally, the more frequent the alcohol intake, the more risk there is of developing alcoholism and chronic damage to organs (Nishiyori et al., 2005).

Studies on the genotype and phenotype of ALDH2 showed that the genotype of ALDH2 was related to the occurrence and development of excess alcohol consumption and alcohol-related diseases (Morimoto and Takeshita, 1996; Chen et al., 1997, 1999), and null ALDH2 activity was found in about 50\% of the Mongolian population (Minami et al., 2002). The Mongolian population lives on the Mongolian plateau in North China with cold climate. They are known to be heavy drinkers. A previous study showed that there is a high incidence of hypertension in Mongolians (Wang, 2005). Alcohol consumption may be the potential factor for the increase in blood pressure. Therefore, we hypothesized that ALDH2 may contribute to the susceptibility to essential hypertension in the Mongolian population from Inner Mongolia. In the present study, we investigated the association between ALDH2 SNP rs671 and EH in Mongolians to provide clues for the prevention of hypertension in Mongolians and data for SNP information in Mongolians. 


\section{MATERIAL AND METHODS}

\section{Subjects}

All subjects gave informed consent to participate in the study. Ninety-one EH patients (44 males and 47 females) with an average age of $51.95 \pm 10.96$ years were enrolled. Blood pressure was measured three times with a sphygmomanometer and the average was recorded. Hypertension was diagnosed according to WHO criteria: systolic blood pressure (SBP) $\geq 140 \mathrm{mmHg}$ and/or diastolic blood pressure (DBP) $\geq 90 \mathrm{mmHg}$, including those who received pharmacologic treatment with antihypertensive drugs in the preceding two weeks. Individuals with secondary hypertension, diabetes mellitus, or severe liver, kidney, and thyroid dysfunction were excluded from the study. Seventy normotensive individuals (37 males and 33 females) with an average age of $44.32 \pm 7.83$ years were enrolled as the control. The normotensive individuals were selected based on SBP $<140 \mathrm{mmHg}$ and/or DBP $<90 \mathrm{mmHg}$, excluding those with antihypertensive medication history, hypertension and other cardiovascular or cerebrovascular diseases. All subjects were from families who had been living in the pastoral area of Inner Mongolia for at least three generations and keeping the traditional nomadic way of life. Our experiment was approved by the Inner Mongolia Medical College Affiliated Hospital Ethics Committee.

\section{DNA isolation and TaqMan polymerase chain reaction (PCR)}

Three milliliters peripheral venous blood was drawn into sodium citrate solution, and genomic DNA was extracted by the Genomic DNA Purification kit (TaKaRa Biotechnology, Dalian, China). Primers and probes were synthesized by the ABI Company (USA). A $25-\mu \mathrm{L}$ reaction mixture including $5 \mu \mathrm{L}$ DNA, $12.5 \mu \mathrm{L}$ TaqMan PCR master mix, $1.25 \mu \mathrm{L}$ primers and probes, $6.25 \mu \mathrm{L}$ deionized water was placed in an ABI7300 real-time PCR instrument (ABI Company, USA). The reaction conditions were as follows: initial denaturation at $95^{\circ} \mathrm{C}$ for 10 min, followed by 40 cycles of denaturation at $92^{\circ} \mathrm{C}$ for $15 \mathrm{~s}$, and annealing and extension at $60^{\circ} \mathrm{C}$ for $90 \mathrm{~s}$. The results were analyzed by the ABI Prism $7300 \mathrm{HT}$ detection system, using the SDS 1.2 software (Applied Biosystems).

\section{Statistical analysis}

Statistical analysis was performed using the SPSS 13.0 software. Data are reported as means \pm standard deviation, and the comparison of frequencies of genotypes and alleles between two groups was performed by the chi-square test.

\section{RESULTS}

\section{Results of TaqMan PCR}

Two primers and one pair of fluorescence-conjugated probes were used in each reaction. The probe of allele $1(\mathrm{G})$, which matched with the wild type was labeled with FAM (color red); the probe of allele 2 (A), which matched with the mutant was labeled with VIC (color green). Only significantly increased red fluorescence indicated the homozygous allele 1 (GG), 
only significantly increased green fluorescence indicated the homozygous allele 2 (AA), and an increase in both colors of fluorescence signals indicated the heterozygous of allele 1 and allele 2 (AG, Figures 1 and 2). AA was not found in the samples.

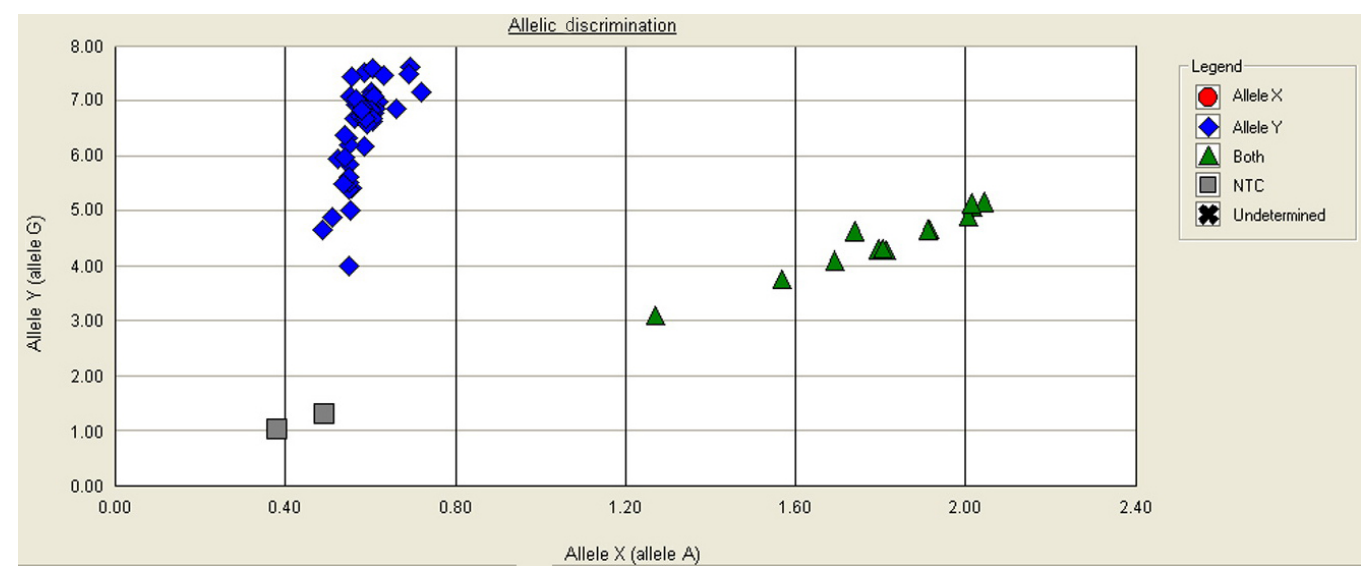

Figure 1. Single nucleotide polymorphism rs671 scatterplot of ALDH2. Allele $\mathrm{X}$ is allele A, shown as a red dot; Allele $\mathrm{Y}$ is allele G, shown as blue diamonds. Both are heterozygous, shown as green triangles; normotensive controls (NTC) are the blanks, shown as gray squares.

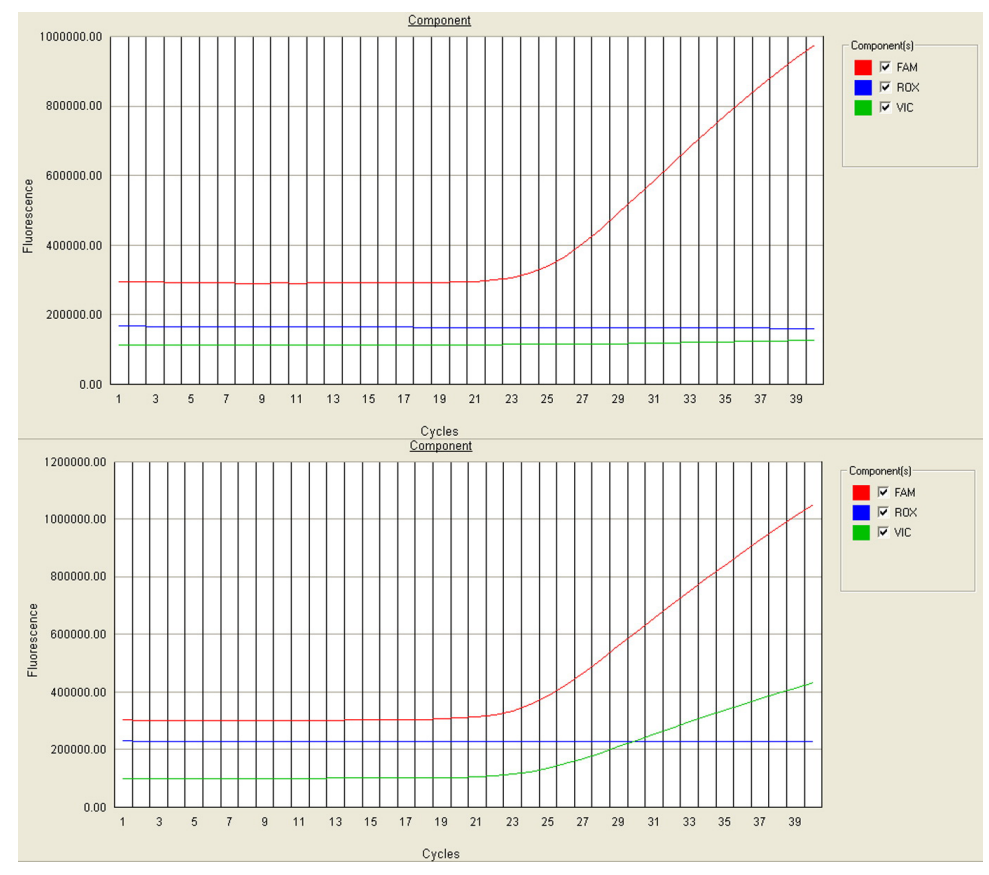

Figure 2. Fluorescence spectrogram of ALDH2 allele G/A in selected tubes during PCR. Only FAM fluorescence (red) increased, indicating homozygous GG (shown in the upper chart). Two fluorescence signals increased, indicating heterozygous AG (shown in the lower chart). ROX fluorescence (blue) was the control. 


\section{ALDH2 SNP rs671 and essential hypertension}

The distribution of ALDH2 SNP rs671 was in accordance with Hardy-Weinberg expectations in the essential hypertension patients and in controls, which showed that the groups studied were representative. Table 1 shows the general information of the subjects. There was no significant difference in gender between the two groups $\left(\chi^{2}=0.321, \mathrm{P}>0.05\right)$.

\begin{tabular}{lcccc}
\multicolumn{5}{l}{ Table 1. General information of the subjects (means \pm standard deviation). } \\
\hline Group & Age (years) & Gender (male/female) & Systolic pressure (mmHg) & Diastolic pressure (mmHg) \\
\hline Control & $44.32 \pm 7.83$ & $37 / 33$ & $116.90 \pm 11.29$ & $76.90 \pm 8.68$ \\
EH & $51.95 \pm 10.96$ & $44 / 47$ & $170.00 \pm 25.27$ & $109.89 \pm 12.93$ \\
\hline
\end{tabular}

$\mathrm{EH}=$ essential hypertension.

The frequencies of the ALDH2*1/1 genotype and ALDH2*1 allele were 91.21 and $95.60 \%$ in patients and 78.57 and $89.29 \%$ in controls, respectively, showing that the frequencies of ALDH $2 * 1 / 1$ genotype and ALDH $2 * 1$ allele in patients were significantly higher than in controls $(\mathrm{P}<0.05$; Table 2$)$. The frequencies of the ALDH $2 * 1 / 2$ genotype and ALDH $2 * 2$ allele were 8.79 and $4.4 \%$ in patients and 21.43 and $10.71 \%$ in controls, respectively, indicating that there were much lower frequencies of ALDH2*1/2 genotype and ALDH2*2 allele in patients than in controls $(\mathrm{P}<0.05$; Table 2$)$. Further analysis showed that the ALDH2*1/1 genotype and ALDH $2 * 1$ allele frequencies in female patients (95.75 and $94.87 \%)$ were higher than in female controls ( 69.97 and $84.85 \%, \mathrm{P}<0.05$; Table 2 ), and that the ALDH $2 * 1 / 2$ genotype and ALDH $2 * 2$ allele frequencies in female patients (4.25 and $2.13 \%$ ) were lower than in female controls ( 30.30 and $15.15 \%, \mathrm{P}<0.05$; Table 2 ), while there was no significant difference in male subjects. ALDH $2 * 2 / 2$ was not found in any of the subjects.

\begin{tabular}{|c|c|c|c|c|c|c|c|c|c|}
\hline \multirow[t]{2}{*}{ Group } & \multirow[t]{2}{*}{$\mathrm{N}$} & \multicolumn{2}{|c|}{ Frequency of genotype (\%) } & \multirow[t]{2}{*}{$\chi^{2}$} & \multirow[t]{2}{*}{$\mathrm{P}$} & \multicolumn{2}{|c|}{ Frequency of allele (\%) } & \multirow[t]{2}{*}{$\chi^{2}$} & \multirow[t]{2}{*}{$P$} \\
\hline & & GG & $\mathrm{AG}$ & & & $\mathrm{G}$ & $\mathrm{A}$ & & \\
\hline Control & 70 & $55(78.57)$ & $15(21.43)$ & 5.160 & 0.023 & $125(89.29)$ & $15(10.71)$ & 4.763 & 0.029 \\
\hline $\mathrm{EH}$ & 91 & $83(91.21)$ & $8(8.79)$ & & & $174(95.60)$ & $8(4.40)$ & & \\
\hline Female control & 33 & $23(69.97)$ & $10(30.30)$ & 10.320 & 0.003 & $56(84.85)$ & $10(15.15)$ & 9.480 & 0.004 \\
\hline Female EH & 47 & $45(95.75)$ & $2(4.25)$ & & & $92(97.87)$ & $2(2.13)$ & & \\
\hline Male control & 37 & $32(86.49)$ & $5(13.51)$ & 0.000 & 1.000 & $69(93.24)$ & $5(6.76)$ & 0.000 & 1.000 \\
\hline Male EH & 44 & $38(86.36)$ & $6(13.64)$ & & & $82(93.18)$ & $6(6.82)$ & & \\
\hline
\end{tabular}

$\mathrm{EH}=$ essential hypertension.

\section{DISCUSSION}

Our study showed that ALDH2 polymorphism was associated with essential hypertension in Mongolians, especially in female Mongolians. ALDH2*2/2 was not found in subjects. Results of the relationship between ALDH2 polymorphism and blood pressure reported by previous studies were controversial. Minami et al. (2002) found that alcohol intake decreased DBP in the ALDH2*1/1 homozygotes, and both SBP and DBP in the ALDH2*1/2 heterozygotes and ALDH2*2/2 homozygotes in the time interval from 0 to $18.3 \mathrm{~h}$, but did not change the 24-h average blood pressure. Amamoto et al. (2002) reported that SBP and DBP tended to decrease in the order of ALDH2*1/1 $>* 1 / 1>* 2 / 2$ in Japanese men. Saito et al. (2003) found that the alcohol-blood pressure relationship was significantly stronger in 
men with ALDH2*1/1 than in men with ALDH $2 * 1 / 2$ or $* 2 / 2$. All studies indicated that the influence of ALDH genotype on blood pressure was complex, depending on the amount of alcohol intake, the time of blood pressure measurement, and environmental and genetic factors. In our study, we investigated the ALDH2 SNP rs671 in Mongolians who are known to be heavy drinkers and found that the ALDH2*1/1 genotype and ALDH2*1 allele frequencies in EH patients were significantly higher, while the ALDH $2 * 1 / 2$ genotype and ALDH $2 * 2$ allele frequencies in $\mathrm{EH}$ patients were much lower than in controls.

Several studies have suggested that ALDH2 polymorphism is a risk factor for hypertension mainly through its association with the level of alcohol consumption. Excluding alcohol intake, there may not be a causal relationship between hypertension and the ALDH2 genotype (Itoh et al., 1997; Takagi et al., 2001; Amamoto et al., 2002; Chen et al., 2008; TsuchihashiMakaya et al., 2009). On the contrary, Hui et al. (2007) reported that the GG (Glu/Glu) genotype of ALDH2 was an independent risk factor for EH. There was no interaction between the ALDH2 genotype and alcohol consumption. In our study, we did not find ALDH2*2/2 in any of the Mongolian subjects, who were free of adverse symptoms, and therefore, the Mongolians could thus drink more alcohol and may have had a greater risk of developing EH. Consistent with our finding, Tsuchihashi-Makaya et al. (2009) found that all the AA homozygotes turned out to be nondrinkers. Our study suggested that ALDH $2 * 2$ was a negative risk factor of $\mathrm{EH}$, and the ALDH2 polymorphism may be a risk factor for EH through alcohol consumption.

All previous studies had found that ALDH2 genotype is a potential risk factor for hypertension among males (Takagi et al., 2001; Amamoto et al., 2002; Hui et al., 2007). Alcohol consumption was less susceptible to blood pressure in females than in males when small amounts of alcohol were consumed ( $0.5 \mathrm{~g} /$ day $)$, while, there were more males with ALDH2*1/1 who drank more alcohol (more than $1 \mathrm{~g}$ /day), and had a greater risk of developing hypertension (Takagi et al., 2001). Our result was opposite to these studies. We found that ALDH2 polymorphism was associated with EH in female Mongolians but not in male Mongolians. The conflict in the results can be explained in part by variations in the amount and type of alcohol intake and cultural tradition of alcohol consumption among female Mongolians. In addition, racial background has also been suggested to play a role.

Taken together, our study found that ALDH2 polymorphism was associated with $\mathrm{EH}$ in Mongolians, especially in female Mongolians. ALDH2*2 was a negative risk factor of EH in Mongolians from Inner Mongolia. Our results address a warning about the drinking behavior of Mongolians.

\section{ACKNOWLEDGMENTS}

We thank all subjects for their participation in the study and Lan Yu for her effort in revising the manuscript. Research supported by the Natural Sciences Fund: Ministry of Education ChunHui Plan.

\section{REFERENCES}

Amamoto K, Okamura T, Tamaki S, Kita Y, et al. (2002). Epidemiologic study of the association of low-Km mitochondrial acetaldehyde dehydrogenase genotypes with blood pressure level and the prevalence of hypertension in a general population. Hypertens. Res. 25: 857-864.

Beilin LJ (1995). Alcohol, hypertension and cardiovascular disease. J. Hypertens. 13: 939-942. 
Chen WJ, Loh EW, Hsu YP and Cheng AT (1997). Alcohol dehydrogenase and aldehyde dehydrogenase genotypes and alcoholism among Taiwanese aborigines. Biol. Psychiatry 41: 703-709.

Chen CC, Lu RB, Chen YC, Wang MF, et al. (1999). Interaction between the functional polymorphisms of the alcoholmetabolism genes in protection against alcoholism. Am. J. Hum. Genet. 65: 795-807.

Chen L, Davey SG, Harbord RM and Lewis SJ (2008). Alcohol intake and blood pressure: a systematic review implementing a Mendelian randomization approach. PLoS. Med. 5: e52.

Ferguson RA and Goldberg DM (1997). Genetic markers of alcohol abuse. Clin. Chim. Acta 257: 199-250.

Hui P, Nakayama T, Morita A, Sato N, et al. (2007). Common single nucleotide polymorphisms in Japanese patients with essential hypertension: aldehyde dehydrogenase 2 gene as a risk factor independent of alcohol consumption. Hypertens. Res. 30: 585-592.

Itoh T, Matsumoto M, Nakamura M, Okada A, et al. (1997). Effects of daily alcohol intake on the blood pressure differ depending on an individual's sensitivity to alcohol: oriental flushing as a sign to stop drinking for health reasons. $J$. Hypertens. 15: 1211-1217.

Minami J, Todoroki M, Ishimitsu T, Yamamoto H, et al. (2002). Effects of alcohol intake on ambulatory blood pressure, heart rate, and heart rate variability in Japanese men with different ALDH2 genotypes. J. Hum. Hypertens. 16: 345-351.

Morimoto K and Takeshita T (1996). Low Km aldehyde dehydrogenase (ALDH2) polymorphism, alcohol-drinking behavior, and chromosome alterations in peripheral lymphocytes. Environ. Health Perspect. 104 (Suppl 3): 563-567.

Nishiyori A, Shibata A, Ogimoto I, Uchimura N, et al. (2005). Alcohol drinking frequency is more directly associated with alcohol use disorder than alcohol metabolizing enzymes among male Japanese. Psychiatry Clin. Neurosci. 59: 38-44.

Saito K, Yokoyama T, Yoshiike N, Date C, et al. (2003). Do the ethanol metabolizing enzymes modify the relationship between alcohol consumption and blood pressure? J. Hypertens. 21: 1097-1105.

Takagi S, Baba S, Iwai N, Fukuda M, et al. (2001). The aldehyde dehydrogenase 2 gene is a risk factor for hypertension in Japanese but does not alter the sensitivity to pressor effects of alcohol: the Suita study. Hypertens. Res. 24: 365-370.

Tsuchihashi-Makaya M, Serizawa M, Yanai K, Katsuya T, et al. (2009). Gene-environmental interaction regarding alcohol-metabolizing enzymes in the Japanese general population. Hypertens. Res. 32: 207-213.

Wang D (2005). Report on Nutrition and Health Survey of Chinese, 2002 Comprehensive Report. People's Medical Publishing House, Beijing,

Yamamoto K, Ueno Y, Mizoi Y and Tatsuno Y (1993). Genetic polymorphism of alcohol and aldehyde dehydrogenase and the effects on alcohol metabolism. Arukoru Kenkyu to Yakubutsu Izon 28: 13-25. 